\title{
A novel general-purpose theorem for the analysis of linear circuits
}

\author{
Ferran Reverter and Manel Gasulla
}

\begin{abstract}
A novel general-purpose theorem for the analysis of linear circuits is stated and proven in this paper. When applying the proposed theorem, any current (voltage) of interest is determined by finding first an equivalent voltage (current) and an equivalent resistance. Although two equivalent parameters have to be found to determine the variable of interest, these are evaluated in circuits that are simpler than the original one, thus resulting in a more straightforward analysis technique. Examples are provided to show the applicability and advantages of the proposed theorem.
\end{abstract}

Index Terms - Circuit theory, circuit analysis, circuit theorem, linear circuits, network theorem.

\section{INTRODUCTION}

$\mathrm{L}$ inear circuits can be analyzed applying different techniques. Two well-stablished and systematic techniques are the node-voltage and the mesh-current methods. However, the analysis can become easier and more intuitive by applying well-known theorems such as superposition, Thévenin, Norton, and maximum power transfer. These theorems were stated more than one hundred years ago, but they are still nowadays the main analysis tools explained in classical university textbooks about circuit analysis [1], [2]. Recently, Thévenin and Norton theorems have been re-explained to show, on the one hand, how powerful they are and, on the other hand, the misconceptions about them [3], [4]. Other theorems for circuit analysis, which are relatively more recent, can be found in the literature, but these are more specific than those indicated before. Some examples are: Millman's theorem [5], Miller's theorem [6], [7], extra-element theorem [8], cut-insertion theorem [9], [10], Foster's theorem [11], and reciprocal power theorem [12].

This paper aims to provide a novel general-purpose theorem for the analysis of linear circuits, complementing the four basic theorems indicated above. The analysis of circuits by means of the proposed theorem involves the determination of an equivalent voltage and an equivalent resistance if the variable of interest is a current, whereas an equivalent current

This work was supported by Universitat Politècnica de Catalunya under the teaching improvement funding program.

F. Reverter and M. Gasulla are with the e-CAT Research Group, Department of Electronic Engineering, Universitat Politècnica de Catalunya (UPC) - BarcelonaTech, C/ Esteve Terradas 7, Castelldefels 08860, Spain (email: ferran.reverter@upc.edu; manel.gasulla@upc.edu). and an equivalent resistance if the variable is a voltage. Although two equivalent parameters have to be determined to know the variable of interest, these are evaluated in circuits that are simpler than the original one and, therefore, the analysis technique is more straightforward. In the following sections, the theorem is stated, two examples are given to show its applicability and advantages, and then the theorem is proven.

\section{THEOREM}

Let us suppose the generic linear circuit represented in Fig. 1a, where the rectangular symbol can be either a passive or an active two-terminal element. Here, we assume resistors and independent voltage/current sources, but we could also have capacitors, inductors, and linearly-controlled sources. The proposed theorem states that:

1. Any current of the circuit (for instance, $I_{\mathrm{A}}$ in Fig. 1a) can be determined as $V_{\text {eqA }} / R_{\text {eqA }}$, where $V_{\text {eqA }}$ and $R_{\text {eqA }}$ are an equivalent voltage and resistance, respectively. In order to calculate these parameters, the current path of $I_{\mathrm{A}}$ must be blocked through an open circuit, as shown in Fig. 1b, and then:

1.1. $R_{\text {eqA }}$ is the resistance between terminals 1 and 2 of the intended open circuit; this resistance must be found by turning off all the independent sources, as usual.

1.2. $V_{\text {eqA }}$ is the open-circuit voltage between terminals 1 and 2. The polarity of $V_{\text {eqA }}$ must be in accordance with the direction of $I_{\mathrm{A}}$.

2. Any voltage of the circuit (for instance, $V_{\mathrm{B}}$ in Fig. 1a) can be determined as $I_{\mathrm{eqB}} \cdot R_{\mathrm{eqB}}$, where $I_{\mathrm{eqB}}$ and $R_{\mathrm{eqB}}$ are an equivalent current and resistance, respectively. These parameters must be calculated as:

2.1. $R_{\text {eqB }}$ is the resistance between the nodes (indicated as terminals 1 and 2 in Fig. 1c) of the voltage difference under study; this resistance is again found by turning off all the independent sources.

2.2. $I_{\text {eqB }}$ is the current flowing between terminals 1 and 2 when these are short-circuited, as shown in Fig. 1d. The direction of $I_{\mathrm{eqB}}$ must be in accordance with the polarity of $V_{\mathrm{B}}$.

This theorem not only provides a new method for the analysis of circuits, but it also has the benefit that calculating $V_{\text {eqA }}$ and $R_{\text {eqA }}$ (or $I_{\text {eqB }}$ and $R_{\text {eqB }}$ ) is expected to be easier than directly calculating $I_{\mathrm{A}}$ (or $V_{\mathrm{B}}$ ). This is because the circuit becomes simpler as a consequence of applying the open circuit (or the short circuit) indicated by the theorem. 
(a)

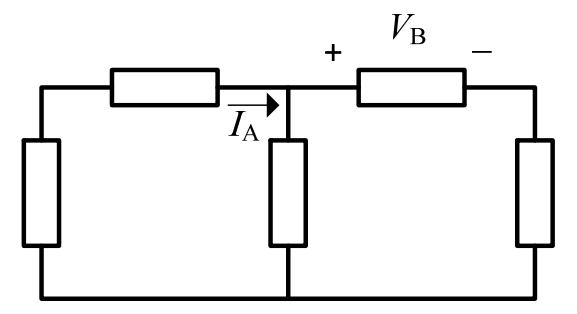

(b)

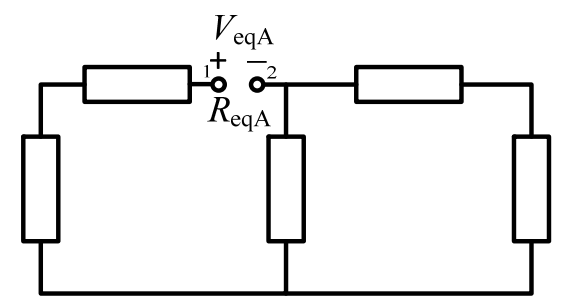

(c)

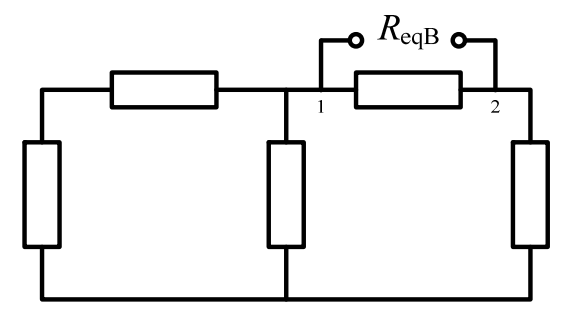

(d)

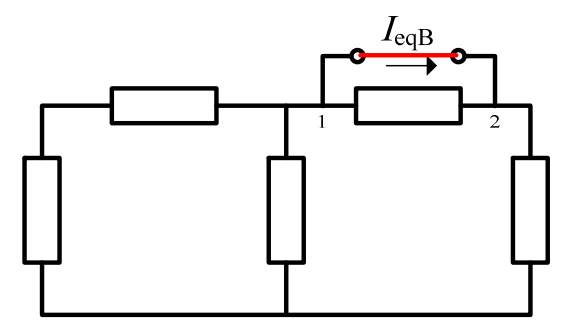

Fig. 1. Generic linear circuit employed to explain how to apply the proposed theorem.

\section{EXAMPLE 1}

Let us suppose the two-mesh circuit shown in Fig. 2, where the current $I_{\mathrm{A}}$ and the voltage $V_{\mathrm{B}}$ have to be found. When applying the proposed theorem to find $I_{\mathrm{A}}$, first the current path has to be blocked through an open circuit. Then, the equivalent resistance and voltage can be determined using the circuits in Figs. 3a and 3b, respectively, as follows:

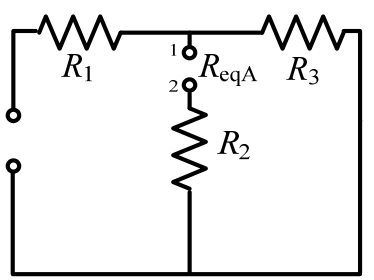

(a)

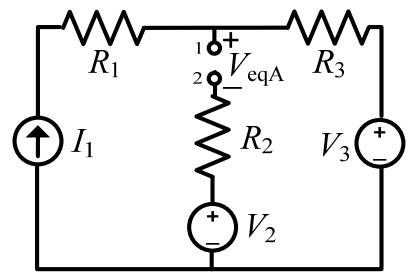

(b)

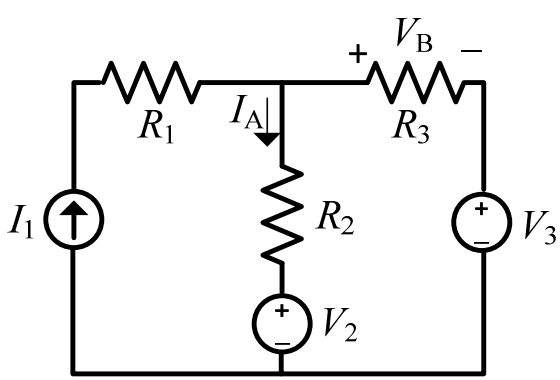

Fig. 2. Two-mesh circuit example where the proposed theorem is applied.

1. $R_{\text {eqA }}$ : The equivalent resistance between terminals 1 and 2 when the sources are turned off is

$$
R_{\text {eqA }}=R_{2}+R_{3}
$$

2. $V_{\text {eqA }}$ : The open-circuit voltage between terminals 1 and 2 can be calculated using, for example, the superposition theorem as

$$
V_{\mathrm{eqA}}=V_{\mathrm{eq}, \mathrm{II}}+V_{\mathrm{eq}, \mathrm{V} 2}+V_{\mathrm{eq}, \mathrm{V} 3}=I_{1} R_{3}-V_{2}+V_{3}
$$

Consequently, from (1) and (2), $I_{\mathrm{A}}$ can be expressed as:

$$
I_{\mathrm{A}}=\frac{V_{\mathrm{eqA}}}{R_{\mathrm{eqA}}}=\frac{I_{1} R_{3}-V_{2}+V_{3}}{R_{2}+R_{3}}
$$

As for the voltage $V_{\mathrm{B}}$ in Fig. 2, the equivalent resistance and current are determined through the circuits in Figs. 3c and 3d, respectively, in the following way:

1. $R_{\text {eqB }}$ : The equivalent resistance between terminals 1 and 2 when the sources are turned off is

$$
R_{\text {eqB }}=R_{2} \| R_{3}
$$

2. $I_{\mathrm{eqB}}$ : The short-circuit current between terminals 1 and 2 can be calculated using, for instance, the superposition theorem as

$$
I_{\mathrm{eqB}}=I_{\mathrm{eq}, \mathrm{Il}}+I_{\mathrm{eq}, \mathrm{V} 2}+I_{\mathrm{eq}, \mathrm{V} 3}=I_{1}+\frac{V_{2}}{R_{2}}-\frac{V_{3}}{R_{2}}
$$

According to (4) and (5), $V_{\mathrm{B}}$ equals

$$
V_{\mathrm{B}}=I_{\text {eqB }} R_{\text {eqB }}=\left(I_{1}+\frac{V_{2}-V_{3}}{R_{2}}\right)\left(R_{2} \| R_{3}\right)
$$

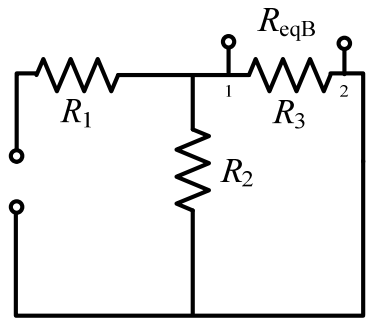

(c)

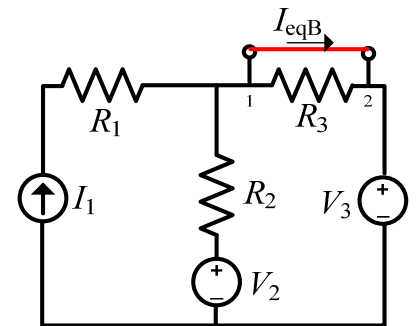

(d)

Fig. 3. Application of the proposed theorem to find $I_{\mathrm{A}}$ and $V_{\mathrm{B}}$ for the example shown in Fig. 2. 


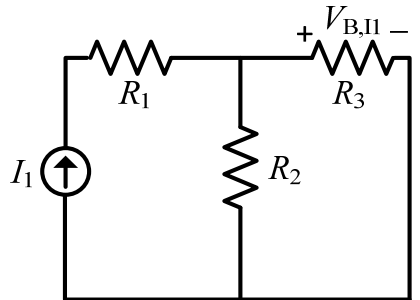

(a)

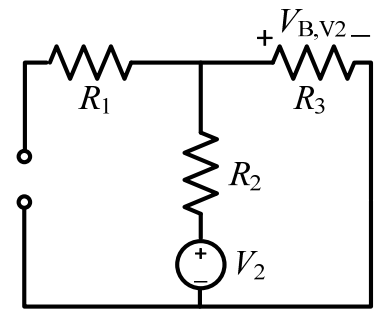

(b)

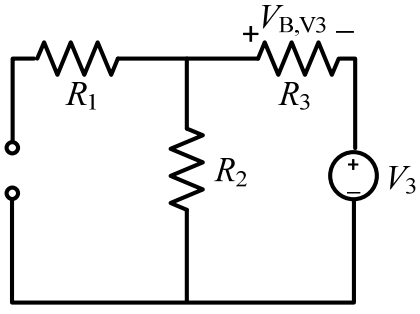

(c)

Fig. 4. Analysis of the circuit in Fig. 2 applying exclusively the superposition theorem.

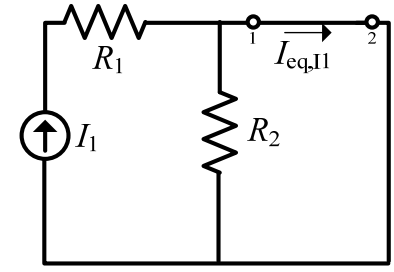

(a)

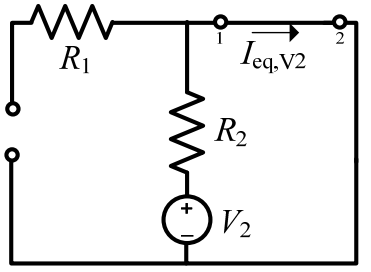

(b)

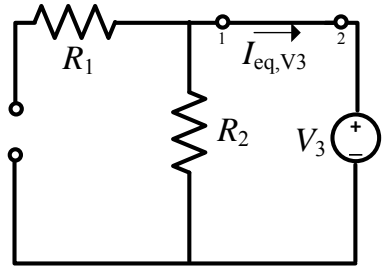

(c)

Fig. 5. Analysis of the circuit in Fig. 3d applying the superposition theorem.

Let us compare the analysis of the circuit in Fig. 2 (for instance, to determine $V_{\mathrm{B}}$ ) without and with the proposed theorem. On the one hand, if the circuit is evaluated using exclusively the superposition theorem, the three subcircuits shown in Fig. 4 are obtained; cases (a), (b), and (c) correspond to the individual effects of $I_{1}, V_{2}$, and $V_{3}$, respectively. In case (a), the circuit behaves as a current divider, whereas in cases (b) and (c), the circuit becomes a voltage divider. On the other hand, if the proposed theorem is employed, we need to find $R_{\text {eqB }}$ and $I_{\text {eqB }}$. For the latter, the resulting subcircuits when applying the superposition theorem in Fig. 3d are shown in Fig. 5. Comparing the circuits in Fig. 4 with those in Fig. 5, one realizes that the voltage/current dividers in Fig. 4 are converted to simpler circuits thanks to the required short circuit that makes $R_{3}$ superfluous. Consequently, obtaining the expression of the three subcomponents of $I_{\mathrm{eqB}}$ is more straightforward than those of $V_{\mathrm{B}}$.

\section{EXAMPLE 2}

The proposed theorem is next applied to a more complex three-mesh circuit represented in Fig. 6, where $I_{\mathrm{A}}$ and $V_{\mathrm{B}}$ have to be found. As for $I_{\mathrm{A}}$, the current path is first blocked via an open circuit, and the equivalent parameters are:

1. $R_{\text {eqA }}$ : Between terminals 1 and 2 , there is an equivalent resistance when the sources are turned off, as shown in Fig. $7 \mathrm{a}$, equal to

$$
R_{\text {eqA }}=R_{1}+R_{2}
$$

2. $V_{\text {eqA }}$ : Between terminals 1 and 2, there is an open-circuit voltage, as shown in Fig. 7b, that can be calculated using, for example, the superposition theorem as

$$
V_{\text {eqA }}=V_{\text {eq, }, 11}+V_{\text {eq, }, 2}+V_{\text {eq, } \mathrm{V} 1}+V_{\text {eq, }, 2}=I_{1} R_{1}+I_{2} R_{2}+0-V_{2}
$$

Therefore, from (7) and (8), $I_{\mathrm{A}}$ is

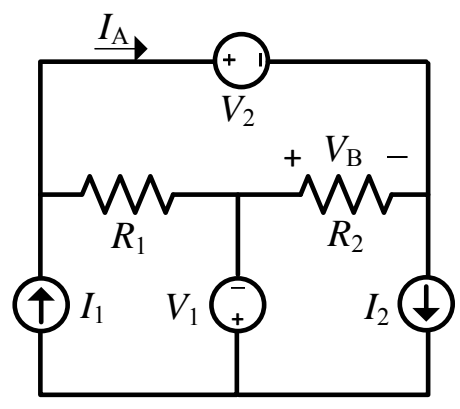

Fig. 6. Three-mesh circuit example where the proposed theorem is applied.

$$
I_{\mathrm{A}}=\frac{V_{\mathrm{eqA}}}{R_{\mathrm{eqA}}}=\frac{I_{1} R_{1}+I_{2} R_{2}-V_{2}}{R_{1}+R_{2}}
$$

On the other hand, the equivalent parameters to determine $V_{\mathrm{B}}$ in Fig. 6 are:

1. $R_{\mathrm{eqB}}$ : Between terminals 1 and 2 , there is an equivalent resistance when the sources are turned off, as shown in Fig. $7 \mathrm{c}$, equal to:

$$
R_{\mathrm{eqB}}=R_{1} \| R_{2}
$$

2. $I_{\text {eqB }}$ : Between terminals 1 and 2 , there is a short-circuit current, as shown in Fig. 7d, that can be calculated using, for instance, the superposition theorem as

$$
I_{\mathrm{eqB}}=I_{\mathrm{eq}, \mathrm{II}}+I_{\mathrm{eq}, \mathrm{I} 2}+I_{\mathrm{eq}, \mathrm{V} 1}+I_{\mathrm{eq}, \mathrm{V} 2}=-I_{1}+I_{2}+0+\frac{V_{2}}{R_{1}}
$$

According to (10) and (11), $V_{\mathrm{B}}$ equals

$$
V_{\mathrm{B}}=I_{\mathrm{eqB}} R_{\mathrm{eqB}}=\left(-I_{1}+I_{2}+\frac{V_{2}}{R_{1}}\right)\left(R_{1} \| R_{2}\right)
$$




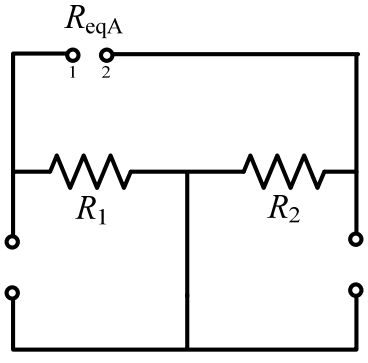

(a)

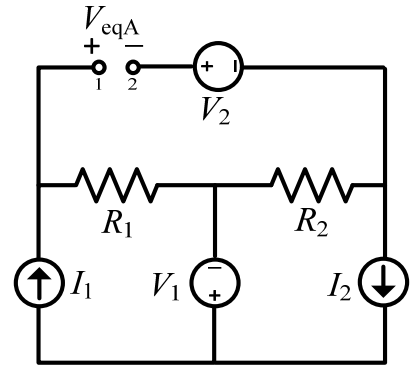

(b)

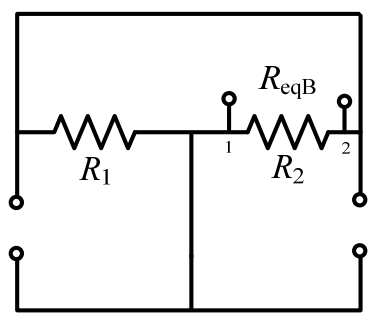

(c)

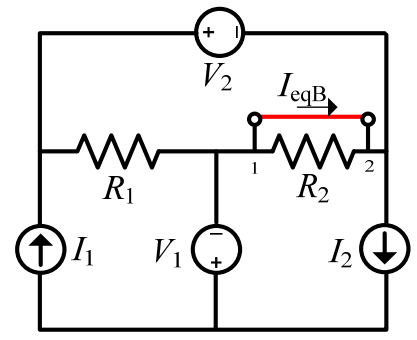

(d)

Fig. 7. Application of the proposed theorem to find $I_{\mathrm{A}}$ and $V_{\mathrm{B}}$ for the example shown in Fig. 6 .

(a)

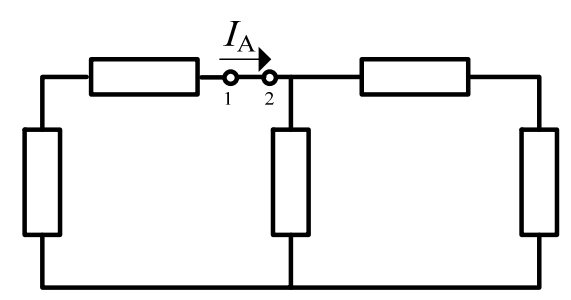

(b)

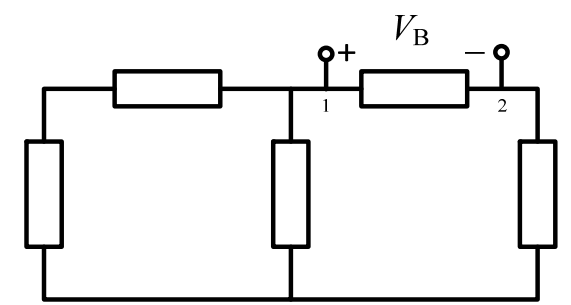

Fig. 8. Proof of the statements by means of the Thévenin and Norton theorems.

\section{PRoof AND Limitations}

The statements in Section II can be proven using the Thévenin and Norton theorems as follows:

Statement \#1: Let us assume the circuit in Fig. 1a where the current $I_{\mathrm{A}}$ is to be determined, but considering two dummy terminals 1 and 2, as shown in Fig. 8a. In such conditions, $I_{\mathrm{A}}$ can be seen as the Norton current of the circuit considering that the "load" was connected between terminals 1 and 2 [1], [2]; in the circuit under analysis, no load will be connected between these terminals, but the Norton theorem is still applicable. Therefore, if $I_{\mathrm{A}}$ is the Norton current, it can also be calculated by means of the Thévenin voltage and resistance, and to do that, terminals 1 and 2 must be left in open circuit in both cases, as stated before and represented in Fig. $1 \mathrm{~b}$.

Statement \#2: Let us consider the circuit in Fig. 1a where the voltage $V_{\mathrm{B}}$ is to be found, but considering again two dummy terminals 1 and 2, as shown in Fig. 8b. Now, $V_{\mathrm{B}}$ can be seen as the Thévenin voltage of the circuit assuming that the "load" was connected between terminals 1 and 2; as indicated before, no load will be connected between these terminals, but the Thévenin theorem is still applicable. Consequently, if $V_{\mathrm{B}}$ is the Thévenin voltage, it can also be calculated through the Norton current and resistance, and to do that, terminals 1 and 2 must be short-circuited and left open, respectively, as stated before and represented in Figs. 1d and $1 \mathrm{c}$.
The proposed theorem not only is proven via Thévenin and Norton theorems, but it also extends the applicability of the concepts behind them to circuits that do not have the classical source-to-load interface [1], [2]. For instance, if $I_{\mathrm{A}}$ has to be determined in the circuit shown in Fig. 1a, the concepts of "source", "load", and "two-terminal interface" are generally not applied. However, by introducing two dummy terminals 1 and 2, as shown in Fig. 8a, the circuit can be seen from a different angle: all the circuit (except for the short circuit between terminals 1 and 2) is the "source", the short circuit between terminals 1 and 2 is the "load", and the two dummy terminals represent the "two-terminal interface". Since the "load" is a short circuit, the load current $\left(I_{\mathrm{A}}\right)$ equals the Norton current. Consequently, this current can also be computed by means of the equivalent resistance and voltage of the Thévenin model, and to find them the "load" must be in open circuit, as proposed herein. The fact of assuming that a short circuit (or an open circuit in Fig. 8b) between two dummy terminals takes the role of the "load" to find $I_{\mathrm{A}}$ (or $V_{\mathrm{B}}$ ) has never been considered before in the literature for the analysis of linear circuits.

After testing the theorem in different linear circuits, the following limitation has been found. If the theorem is applied to find the voltage across the terminals of a voltage source or the current in series with a current source, the result has an indeterminate form although the response is straightforward without using any analysis technique. Actually, the Thévenin (Norton) theorem has the same limitation when it is applied to a circuit with just an ideal current (voltage) source.

\section{CONCLUSION}

With the aim of complementing the basic theorems for circuit analysis that were stated more than one hundred years ago, a novel general-purpose theorem has been stated and proven herein. When applying this theorem, the circuit is looked from a different angle: any current (voltage) of interest is determined by finding an equivalent voltage (current) and an equivalent resistance. Since these two equivalent parameters are evaluated in circuits that are simpler than the original one, the analysis becomes more straightforward. In the opinion of the authors, the statements are easy to remember and to apply and, hence, the proposed theorem is expected to become a basic tool in the circuit theory. 


\section{REFERENCES}

[1] W. Hayt, J. Kemmerly, J. Phillips, and S. Durbin, Engineering Circuit Analysis, 9th ed. New York: McGrawHill, 2019.

[2] R.E. Thomas, A.J. Rosa, and G.J. Toussaint, The Analysis and Design of Linear Circuits, 9th ed. Hoboken, NJ: Wiley, 2019.

[3] A. Sheikholeslami, "Thevenin and Norton Equivalent Circuits: Part 1," IEEE Solid-State Circuits Mag., vol. 10, no. 2, pp. 8-10, Mar. 2018.

[4] A. Sheikholeslami, "Thevenin and Norton Equivalent Circuits: Part 2," IEEE Solid-State Circuits Mag., vol. 10, no. 3, Jun. 2018.

[5] J. Millman, "A Useful Network Theorem," Proc. IRE, vol. 28, no. 9, pp. 413-417, 1940.

[6] M. D. Davidović, “A simple proof of Miller's theorem," IEEE Trans. Educ., vol. 42, no. 2, pp. 154-155, 1999.

[7] A. Sheikholeslami, "Miller's Theorem," IEEE Solid-State Circuits Mag., vol. 7, no. 3, pp. 9-10, Jun. 2015.

[8] R. D. Middlebrook, "Null Double Injection and the Extra Element Theorem," IEEE Trans. Educ., vol. 32, no. 3, pp. 167-180, 1989.

[9] B. Pellegrini, "Improved feedback theory," IEEE Trans. Circuits Syst. I Regul. Pap., vol. 56, no. 9, pp. 1949-1959, 2009.

[10] I. M. Filanovsky, "Critical analysis of cut-insertion theorem," in Midwest Symposium on Circuits and Systems, 2012, pp. 658-661.

[11] K. Thulasiraman, M. Yadav, and K. Naik, "Network science meets circuit theory: Resistance distance, Kirchhoff index, and Foster's theorems with generalizations and unification," IEEE Trans. Circuits Syst. I Regul. Pap., vol. 66, no. 3, pp. 1090-1103, Mar. 2019.

[12] F. Broyde and E. Clavelier, "Two Reciprocal Power Theorems for Passive Linear Time-Invariant Multiports," IEEE Trans. Circuits Syst. I Regul. Pap., vol. 67, no. 1, pp. 86-97, Jan. 2020. 